\title{
On stabilization of discrete-event processes
}

\author{
Y. BRAVE ${ }^{\dagger}$ and M. HEYMANN ${ }^{\ddagger}$
}

Discrete-Event processes are modeled by state-machines in the Ramadge-Wonham framework with control by a feedback event-disablement mechanism. In this paper concepts of stabilization of discrete-event processes are defined and investigated. We examine the possibility of driving a process (under control) from arbitrary initial states to a prescribed subset of the state set and then keeping it there indefinitely. This stabilization property is studied also with respect to 'open-loop' processes (i.e., uncontrolled processes) and their asymptotic behavior is characterized. To this end, such well known classical concepts of dynamics as invariant-sets and attractors are redefined and characterized in the discrete-event control framework. Finally, we provide polynomial time algorithms for verifying various types of attraction and for the synthesis of attractors.

\section{Introduction}

This paper is a preliminary investigation of the concepts of stabilization of discrete-event processes (DEP). We adopt a slightly modified version of the framework proposed by Ramadge and Wonham (1987 a, 1987 b, Wonham and Ramadge 1987) for the study of DEP. Our model is thus a state machine with a means of external control: a feedback event-disablement mechanism. Unlike Ostroff and Wonham 1987, Brave and Heymann 1989, Golaszewski and Ramadge 1989, we consider a state model describing the possible order of elementary events but not their exact timing.

In most of the works concerning supervisory control of DEP (e.g., Cieslak et al. 1988, Cho and Marcus 1987, Lin and Wonham 1987, Yong and Wonham 1988) it is assumed that the initial

\footnotetext{
${ }^{\dagger}$ Department of Electrical Engineering, Technion - Israel Institute of Technology, Haifa 32000 , Israel.

${ }^{\ddagger}$ NRC Senior Research Associate, NASA-Ames Research Center, Moffett Field, California 94035, on leave from Department of Computer Science, Technion - Israel Institute of Technology Haifa 32000 , Israel.
} 
state of the process is fixed, known apriori and one of the 'legal' states of the process. The control problem is then to synthesize a supervisor which confines the behavior of the process, initialized at the prespecified initial state, to within legal bounds. However, there are cases in which either the initial state is not one of the legal states of the process or it is unknown apriori. In such cases the question of stabilization is of great interest.

In this paper we study the ability of a process to reach a set of target states from an arbitrary initial state and then remain there indefinitely. This stabilization property is examined under different control strategies. To this end, the classical concept of attraction (Bhatia and Szego 1967) is reformulated and characterized in our framework. Polynomial time algorithms are provided for the verification of different types of attraction.

This paper is organized as follows. In the remainder of this section we give some terminology and notation. Invariant sets of states and realizable processes are defined in section 2. In section 3, the notion of strong attraction is introduced and examined with respect to processes without external control. Further, an efficient algorithm for computing the asymptotic behavior of such processes is proposed. Section 4 develops control strategies under which strong attraction can be achieved. To this end, a weaker form of attraction is introduced. An efficient algorithm for computing the region of weak attraction is provided in section 5. In section 6 , an illustrative example is given and the relation between attraction and recovery of failure is mentioned.

\subsection{Processes}

Let $\Sigma$ be a finite alphabet (event set). A process over $\Sigma$ is modeled as a finite (directed) graph $G=(V, E)$ where $V$ is a set of states (vertices) and $E \subseteq V \times \Sigma \times V$ is a set of edges. An edge of $G$ is thus an ordered triple $e=(v, \sigma, u) \in E$ and it is said to be directed from $v$ to $u$. The state $v$ is called the start-state of $e$, the state $u$ is called the end-state of $e$ and $\sigma \in \Sigma$ is the event associated 
with $e$. If $(v, \sigma, u) \in E$ we say that $v$ is a predecessor of $u$ and $u$ is a successor of $v$. Edges with the same start state and the same end state are called parallel. It is assumed that there are no two edges going out of the same state associated with the same event, that is, for each pair of edges in $E$

$$
[(v, \alpha, u),(v, \beta, w) \in E \text { and } \quad \alpha=\beta] \quad \text { implies } \quad u=w .
$$

We interpret $G$ as a device that starts its execution at an arbitrary state $v \in V$ ( $v$ may be determined by a nondeterministic mechanism in $G$ or forced externally) and thereafter executes a sequence of state transitions as permitted by $E$.

A path is a finite sequence of edges $e_{1}, e_{2}, \ldots, e_{n}$ such that the end state of $e_{i-1}$ is the start state of $e_{i}$. The number of edges in a path is called the length of a path. The start of the path is the start state of $e_{1}$ and its end is the end state of $e_{n}$. To each path $\left(v_{0}, \sigma_{1}, v_{1}\right),\left(v_{1}, \sigma_{2}, v_{2}\right), \ldots,\left(v_{n-1}, \sigma_{n}, v_{n}\right)$ there corresponds a unique (state) trajectory $v_{0}, v_{1}, \ldots, v_{n}$. Further, if $v_{0}=v_{n}$ the trajectory is said to be closed. A closed trajectory in which no state (except the start and end states) appears more than once is called a cycle. A graph without cycles is called acyclic.

A state $v$ is reachable from a state $u$ if there exists a path from $u$ to $v$. A state $v$ is said to be reachable from a subset of states $A$ if $v$ is reachable from at least one state in $A$. The reach of $A$ in $G$, denoted $r_{G}(A)$, is defined as the set of all states in $G$ that are reachable from $A$.

Let $G=(V, E), \varnothing \neq A \subseteq V$. We say that a state $v \in V-A$ is connected to $A$ if there is a path from $v$ to a state in $A$. Further, $G$ is called $A$-connected if each $v \in V-A$ is connected to $A$. A process $G^{\prime}=\left(V^{\prime}, E^{\prime}\right)$ is called a subprocess of the process $G=(V, E)$, denoted $G^{\prime} \subseteq G$, if $V^{\prime} \subseteq V$ and $E^{\prime} \subseteq E$.

The union of two processes $G_{1}=\left(V_{1}, E_{1}\right)$ and $G_{2}=\left(V_{2}, E_{2}\right)$ is another process $G_{3}$ (written $G_{3}=G_{1} \cup G_{2}$ ) whose state set is $V_{3}=V_{1} \cup V_{2}$ and whose edge set is $E_{3}=E_{1} \cup E_{2}$. If $v$ is a state in $G$ then $G-v$ denotes the subprocess of $G$ obtained by deleting $v$ from $G$. Deletion of a 
state always implies the deletion of all edges incident on that state. If $e$ is an edge in $G$, then $G-e$ is a subprocess of $G$ obtained by deleting $e$ from $G$. Deletion of an edge does not imply deletion of its end states.

A subprocess $G^{\prime}=\left(V^{\prime}, E^{\prime}\right) \subseteq G=(V, E)$ is called an induced subprocess if $E^{\prime}$ contains all the edges of $E$ whose end points are in $V^{\prime}$; in this case we say that $G^{\prime}$ is induced by $V^{\prime}$. The process induced by $V^{\prime}$ is denoted $\left\langle V^{\prime}\right\rangle_{G}$.

\subsection{Supervisors}

As in Ramadge and Wonham 1987 a, we assume that $\Sigma$ consists of two disjoint subsets $\Sigma_{u}$ and $\Sigma_{c}$ : uncontrolled and controlled events. Events in $\Sigma_{c}$ can be disabled by external control while events in $\Sigma_{u}$ cannot be prevented from occurring. Clearly, this classification of $\Sigma$ induces a similar classification of $E$, that is, $E=E_{u} \cup E_{c}$ where $E_{u}=E \cap\left(V \times \Sigma_{u} \times V\right)$ and $E_{c}=E-E_{u}$.

A supervisor for $G$ is a map $S: V \rightarrow 2^{\Sigma_{c}}$. For each state $v \in V$ the supervisor specifies a subset of controlled events that must be disabled.

The concurrent operation of the process $G$ and a supervisor $S$, denoted $(S / G)$ and called the closed-loop process, is defined as the subprocess $\left(V, E^{S}\right)$ of $(V, E)$ satisfying the condition that for all $e=(v, \sigma, u) \in E$

$$
e \in E^{S} \quad \text { iff } \quad \sigma \notin S(v)
$$

\section{Invariant Sets of States and Realizable Processes}

Let $G=(V, E)$ be a process and let $A \subseteq V, E^{\prime} \subseteq E$. We say that $A$ is $E^{\prime}$-invariant iff

$$
\left(\forall(v, \sigma, u) \in E^{\prime}\right) \quad v \in A \Rightarrow u \in A .
$$

That is, there is no edge in $E^{\prime}$ leading out of $A$. We remark that the important special case where $A$ is $E_{u}$-invariant has been discussed in Ramadge and Wonham $1987 \mathrm{~b}$, in connection with a 
modular-approach solution for the problem of maintaining a predicate on $V$ invariant.

A subprocess $G^{\prime}=\left(V^{\prime}, E^{\prime}\right)$ of the process $G=(V, E)$ is called realizable iff

$$
\left(\forall(v, \sigma, u) \in E_{u}\right) \quad v \in V^{\prime} \Rightarrow(v, \sigma, u) \in E^{\prime}
$$

That is, a subprocess $G^{\prime} \subseteq G$ is realizable iff every uncontrolled edge going out of a state in $G^{\prime}$ is an edge of $G^{\prime}$. Moreover, it is easily seen that a subprocess $G^{\prime}=\left(V^{\prime}, E^{\prime}\right)$ is realizable iff there exists a supervisor $S$ such that the closed-loop process $(S / G)$ and the subprocess $G^{\prime}$ have the same 'behavior' in the sense that for each state $v \in V^{\prime}$, the set of all paths starting at $v$ is the same in $G^{\prime}$ and $(S / G)$. In fact, the notion of a realizable subprocess is closely related to the concept of controllable language (Ramadge and Wonham 1987 a).

\section{Strong Attraction}

In this section we examine some properties of 'open-loop' processes, i.e., processes without external control. First we introduce the concept of strong attraction.

\subsection{Strong attractors}

Let $G=(V, E)$ be a process and let $A, B \subseteq V$ such that $\varnothing \neq A \subseteq B$. We say that $A$ is a

$G$ strong attractor for $B$ w.r.t. $G$, denoted $A \Leftarrow B$, iff the following conditions are satisfied:

(a1) $A$ is $E$-invariant.

(a2) for each state $v \in r_{G}(B)$ there is a path that starts at $v$ and ends in $A$.

(a3) there are no cycles of $G$ in $r_{G}(B)-A$.

$G$

Thus, if $A \Leftarrow B$ then whenever the process $G$ is initialized at state $v \in B$ it always reaches $A$ within a finite number of state transitions and remains in $A$.

We show now that for each nonempty $E$-invariant subset $A$ of $V$ there exists a unique largest subset for which $A$ is a strong attractor. To this end, let $\varnothing \neq A \subseteq V$ be $E$-invariant, and define 
$T_{G}(A)$ to be the (finite) class of all subsets of $V$ for which $A$ is a strong attractor, that is,

$$
T_{G}(A)=\{B \subseteq V \mid A \subseteq B \quad \text { and } \quad A \stackrel{G}{\models} B\}
$$

\section{Proposition 3.1}

The class $T_{G}(A)$ is nonempty and closed under set union.

\section{Proof}

Nonemptiness is immediate since $A \in T_{G}(A)$. To prove closure under set union let $B_{1}, B_{2} \in T_{G}(A)$, and note that

$$
r_{G}\left(B_{1} \cup B_{2}\right)=r_{G}\left(B_{1}\right) \cup r_{G}\left(B_{2}\right),
$$

whence $B_{1} \cup B_{2}$ satisfies condition (a2). To see that condition (a3) holds with respect to $B_{1} \cup B_{2}$ assume to the contrary that $r_{G}\left(B_{1} \cup B_{2}\right)-A$ contains a cycle $C=v_{1}, v_{2}, \cdots, v_{k}, v_{k}=v_{1}$. Since condition (a3) is satisfied w.r.t. $B_{1}$ and $B_{2}$ separately, it follows that there must exist states $v_{i}$ and $v_{i+1}$ such that

$$
v_{i} \in r_{G}\left(B_{1}\right)-A \text { and } v_{i+1} \notin r_{G}\left(B_{1}\right)-A
$$

However, (3.1) is impossible since there is an edge from $v_{i}$ to $v_{i+1}$, and condition (a3) is satisfied with respect to $\left(B_{1} \cup B_{2}\right)$. This concludes the proof.

An immediate consequence of proposition 3.1 is that $T_{G}(A)$ has a unique maximal element. $G$ The maximal set $B$ for which $A \stackrel{G}{\Leftarrow} B$ is denoted $\Lambda_{G}(A)$ and called the region of strong attraction of $A$ w.r.t. $G$. For a subset $A$ which is not $E$-invariant we will say that $\Lambda_{G}(A)=\varnothing$. If $\Lambda_{G}(A)=V$ we say that $A$ is a global strong attractor w.r.t. $G$ (denoted $A \stackrel{G}{\models}$ ). In cases of no confusion we shall not mention the underlying process and write, e.g., that $A$ is a global strong attractor. It is readily verified that in the case of global strong attraction conditions (a2) and (a3) can be written 
(a2') $\quad G$ is $A$-connected.

(a3') $\quad G-A$ is acyclic.

\subsection{Asymptotic behavior}

The meaning of a subset $A \subseteq V$ being a global strong attractor is that there exists a number

$N \leq|V-A|$ such that every trajectory of $G$ of length greater than $N$ ends in $A$. Further, the subset $A$ is reachable from each state in $V$. In other words, initializing the process at an arbitrary state $v \in V$ causes the process to reach a state in $A$ in a finite number of state transitions. Once the process reaches a state in $A$ it remains in $A$.

A natural question that arises is whether we can maximally restrict the state domain in which the process, initialized at an arbitrary state, can be 'found' after a sufficient large number (bounded by $|V|$ ) of state transitions. That is, we are interested in the asymptotic 'behavior' of the process.

Thus, let $G=(V, E)$ and let $g(G)$ be the (finite) class of all subsets of $V$ that are global strong attractors w.r.t. G. That is,

$$
g(G)=\{A \subseteq V \mid A \stackrel{G}{\models}\}
$$

First we need the following obvious observations.

\section{Observation 3.2}

The state set of $G$ is a global strong attractor (w.r.t. $G$ ). 


\section{Observation 3.3}

Let $C$ be a cycle of $G$. Then $C$ is a cycle of $\langle A\rangle_{G}$ for every $A \in g(G)$.

\section{Proof}

An immediate consequence of conditions (a1) and (a3').

The following proposition states that $g(G)$ is nonempty, closed under set intersection and no two subsets of $g(G)$ are disjoint.

\section{Proposition 3.4}

Let $G=(V, E)$. Then $g(G) \neq \varnothing$, and if $A_{1}, A_{2} \in g(G)$ then

$$
\varnothing \neq A_{1} \cap A_{2} \in g(G) .
$$

\section{Proof}

Clearly, the nonemptiness of $g(G)$ follows from observation 3.2. Let $A_{1}, A_{2} \in g(G)$. Since $G$ is $A_{1}$-connected (condition (a2') applied to $A_{1}$ ), the subset $A_{2}$ cannot be $E$-invariant if $A_{1} \cap A_{2}=\varnothing$. Thus, $A_{1}$ and $A_{2}$ are not disjoint. We shall show now that $A_{1} \cap A_{2}$ is a global strong attractor, namely conditions (a1), (a2') and (a3') are satisfied with respect to $A_{1} \cap A_{2}$.

Condition (a1) is immediate since $A_{1}$ and $A_{2}$ are both $E$-invariant. Next we have to verify condition (a2'), namely that for every state $v \in V$ there is a path which starts at $v$ and ends in $A_{1} \cap A_{2}$. To this end, assume that for some state $v_{o} \in V$ there is no path from $v_{o}$ to $A_{1} \cap A_{2}$. Since $A_{1} \stackrel{G}{\Leftarrow}$, there exists a path $s_{o}$ from $v_{o}$ to $A_{1}$, say to $v_{1} \in A_{1}$. By the last assumption it is clear that $v_{1} \notin A_{2}$. Similarly, $A_{2} \stackrel{G}{=}$ implies the existence of a path $s_{1}$ from $v_{1}$ to a state in $A_{2}$, say $v_{2}$. Since $A_{1}$ is $E$-invariant, every path that starts in $A_{1}$ ends in $A_{1}$. In particular, it is true with respect to $s_{1}$, and thus $v_{2} \in A_{1} \cap A_{2}$. So we conclude that the path $s_{o} s_{1}$ connects $v_{0}$ 
to $A_{1} \cap A_{2}$, a contradiction.

Finally, observation 3.3 implies condition (a3'), namely that $G-\left(A_{1} \cap A_{2}\right)$ is acyclic.

Proposition 3.4 implies that the finite class $g(G)$ contains a unique infimal element w.r.t. inclusion, which is denoted $\inf [g(G)]$. Further, this infimal element satisfies the condition that

$$
\inf [g(G)]=\cap\{A \mid A \in g(G)\}
$$

For an effective computation (i.e., a polynomial time algorithm) of the minimal global strong attractor we need the following proposition.

\section{Proposition 3.5}

Let $G=(V, E)$ and $v \in V$. Then $v \in \inf [g(G)]$ if and only if either

(i) $\quad v$ is reachable from a state of a cycle in $G$; or

(ii) $v$ has no successors.

\section{Proof}

For abbreviation let $W \triangleq \inf [g(G)]$.

(If). Clearly, every global strong attractor of $G$ contains all the states in $G$ which are 'dead-end', namely without outgoing edges. Otherwise, condition (a2') cannot be satisfied. Thus condition (ii) is a sufficient one. As regards condition (i), we note that conditions (a1) and (a3') imply that every cycle of $G$ is contained in every global strong attractor. Moreover, since every global strong attractor $A$ is $E$-invariant it follows that every state reachable from a state in $A$ must be also in $A$. So we conclude that every state reachable from a state of a cycle in $G$ is in $W$, which is one of the global strong attractors of $G$. 
(Only if). Fix $v_{o} \in W$ and suppose, towards a contradiction, that $v_{o}$ does not satisfy conditions (i) and (ii), that is,

(iii) $v_{o}$ has at least one successor ; and

(iv) the subset $X$ of all states in $V$ from which $v_{o}$ is reachable satisfies the condition that every state in $X$ is not a state of a cycle in $G$.

We shall show now that $W-X$ is a global strong attractor, contradicting our assumption that $W$ is the minimal one.

Let $Y$ be the set of all states in $W$ from which $v_{o}$ is reachable, i.e., $Y=W \cap X$. Clearly, $Y$ is not empty (since $v_{o} \in W \cap X=Y$ ) and $W-X=W-Y$. First we claim that $W-Y$ is $E$-invariant. To see this, suppose $W-Y$ is not $E$-invariant, and that for some $u \in W-Y$ there exists an edge $(u, \sigma, w) \in E$ such that $w \notin W-Y$. By the definition of $W$ it is clear that $W \stackrel{G}{\Leftarrow}$ and thus the $E$-invariance of $W$ implies $w \in W$. Since

$$
(w \in W \text { and } w \notin W-Y \text { ) implies } \quad w \in Y \subseteq X \text {, }
$$

it follows that $v_{o}$ is reachable from $w$. Consequently, $(u, \sigma, w) \in E$ implies that $v_{o}$ is reachable also from $u$, i.e., $u \in Y$, contradicting our assumption that $u \in W-Y$. So $W-Y$ is $E$-invariant.

Next we have to show that $G$ is $(W-Y)$-connected, that is, there exists a path from each state in $V-(W-Y)$ to a state in $(W-Y)$. First we consider the state $v_{o}$. Since $v_{o} \in Y \subseteq W$ and $W$ is $E$-invariant then every successor $v_{1}$ of $v_{o}$ is in $W$ (by assumption (iii), $v_{o}$ has at least one successor). Further, $v_{1} \notin Y \subseteq X$ since otherwise $v_{o}$ is reachable from $v_{1}$, meaning that $v_{o}$ is a state of a cycle, in contradiction to assumption (iv). Thus, $v_{1} \in W-Y$ and $v_{o}$ is connected to $(W-Y)$. Moreover, by the definition of $X, v_{o}$ is reachable from every state in $X$ and thus each state in $X$ is connected to $(W-Y)$. Finally, $W$ is a global strong attractor and thus, by condition (a2'), every state in $V-W$ is connected either to $(W-Y)$ or to $Y \subseteq X$, which is connected to $W-Y$. 
It remains to be shown that $G-(W-Y)$ is acyclic, namely that every cycle of $G$ intersects $(W-Y)$. To this end, let $C$ be a cycle of $G$. Since $G-W$ is acyclic and $W$ is $E$-invariant then $C$ is contained in $W$. Further, by assumption (iv), every state in $Y \subseteq X$ is not a state of $C$ and thus $C$ must be contained in $(W-Y)$. That is, $G-(W-Y)$ is acyclic.

To summarize, we have showed that $W-Y$ is also a global strong attractor w.r.t. $G$, contradicting our assumption that $W=\inf [g(G)]$.

Using proposition 3.3 and the transitive closure of $G$ (i.e., the directed graph in which there is an edge from $v$ to $u$ iff there is a nonempty path from $v$ to $u$ in $G$ (Even 1979, Ch. 1)), the infimal global strong attractor $\inf [g(G)]$ can be computed in polynomial time.

\section{Weak Attraction}

In this section we introduce a weaker form of attraction which can be obtained under a suitable control.

\subsection{Weak attractors}

Let $G=(V, E), \varnothing \neq A \subseteq B \subseteq V$. The subset $A$ is called a weak attractor for $B$ w.r.t. $G$, denoted $A \stackrel{G}{\leftarrow} B$, iff there exists a supervisor $S$ such that $A \stackrel{(S / G)}{\Leftarrow} B$.

Clearly, strong attraction implies weak attraction but the converse is in general not true. Further, it is easily seen that a necessary condition for a subset $A$ to be a weak attractor for another subset is that $A$ be $E_{u}$-invariant.

Necessary and sufficient conditions for an $E_{u}$-invariant subset $A$ to be a weak attractor for $B$ are given by the following proposition. 


\section{Proposition 4.1}

$G$

Let $G=(V, E), \varnothing \neq A \subseteq B \subseteq V$, such that $A$ is $E_{u}$-invariant. Then $A \leftarrow B$ if and only if there exists a subprocess $G^{\prime}=\left(V^{\prime}, E^{\prime}\right)$ of $G$ such that $B \subseteq V^{\prime}$ and the following conditions are satisfied:

(b1) $G^{\prime}$ is $A$-connected.

(b2) $G^{\prime}$ is realizable.

(b3) $G^{\prime}-A$ is acyclic.

\section{Corollary 4.1}

If $A$ is $E_{u}$-invariant and $G^{\prime}=\left(V^{\prime}, E^{\prime}\right)$ satisfies condition (b1)-(b3) then $A \stackrel{G}{\leftarrow} V^{\prime}$.

\section{Proof of proposition 4.1}

(If). Let $G^{\prime}=\left(V^{\prime}, E^{\prime}\right) \subseteq G$ such that $B \subseteq V^{\prime}$ and (b1)-(b3) are satisfied. First we shall show that $G^{\prime}$ contains a realizable subprocess $P$ for which $A$ is a global strong attractor. Then we shall construct from $P$ a supervisor as required for proving weak attraction.

The subprocess $P \triangleq(V, D)$ is obtained from $G^{\prime}$ by removing all the controlled edges leaving $A$, that is,

$$
P=G^{\prime}-\left\{(v, \sigma, u) \in E_{c}{ }^{\prime} \mid v \in A \quad \text { and } u \notin A\right\}
$$

Notice that for every set of edges $D$, the notation $D_{c}$ stands for the subset of all controlled edges in $D$, while $D_{u}$ denotes the subset of all uncontrolled edges in $D$.

In order to show that $A \stackrel{P}{\rightleftharpoons}$ we have to verify conditions (a1), (a2') and (a3'). Indeed, since $A \subseteq B \subseteq V^{\prime}$ it follows from (4.1) that $A$ is $D_{c}$-invariant. Further, since by the hypothesis of proposition 4.1, $A$ is $E_{u}$-invariant, the fact that $P$ is a subprocess of $G$ implies that $A$ is also $D_{u^{-}}$ 
invariant. The two last conclusions imply that $A$ is $D$-invariant, establishing condition (a1).

As regards condition (a2'), since $G^{\prime}$ is $A$-connected and $P$ is constructed from $G^{\prime}$ by only deleting edges leaving $A$, it follows that $P$ is also $A$-connected.

Finally, $P-A$ is a subprocess of $G^{\prime}-A$ and thus the fact that $G^{\prime}-A$ is acyclic implies that $P-A$ is also acyclic, satisfying condition (a3').

So we conclude that $A \stackrel{P}{\Leftarrow}$. Moreover, the process $P$ results from $G^{\prime}$ by a deletion of controlled edges and thus the realizability of $P$ follows from the realizability of $G^{\prime}$.

Based on the realizable subprocess $P$, a supervisor $S$ is defined as follows. Let $S$ be a supervisor such that the closed-loop process $(S / G) \triangleq\left(V, E^{S}\right)$ satisfies the condition that $E^{S}=E_{u} \cup D$. That is, the state set of the closed-loop process $(S / G)$ is $V$ (i.e., the state set of $G$ ) while its edge set is the union of the uncontrolled edges of $G$ and the edges of $P$. Clearly, such a supervisor exists since $E^{S}$ contains all the uncontrolled edges of $G$.

Now we claim that $P$ and $(S / G)$ coincide in their behavior in the sense that for every state $v \in V^{\prime}$, the set of all possible paths starting at $v$ is the same in $P$ and $(S / G)$. This property follows from the following observations. The first one is that $P$ is realizable and thus

$$
\left(\forall(v, \sigma, u) \in E_{u}\right) \quad v \in V^{\prime} \quad \text { implies } \quad(v, \sigma, u) \in D \text {. }
$$

That is, every uncontrolled edge in $E$ emanating from a state $v \in V^{\prime}$ is also an edge of $P$. The second observation is that every controlled edge of $G$ is an edge of $(S / G)$ iff it is an edge of $P$. Combining these two observations leads to the conclusion that for all $e=(v, \sigma, u) \in E$ such that $v \in V^{\prime}$,

$$
e \in E^{S} \quad \text { iff } \quad e \in D .
$$

An immediate consequence of (4.2) is that the reach of $V^{\prime}$ in the closed-loop process $(S / G)$ is $V^{\prime}$, i.e., 


$$
r_{(S / G)}\left(V^{\prime}\right)=V^{\prime}
$$

and thus it is readily verified that

$$
A \stackrel{P}{\Leftarrow} \quad \text { implies } \quad A \stackrel{(S / G)}{\Leftarrow} V^{\prime} .
$$

Since $B \subseteq V^{\prime}$ it follows that $A \stackrel{(S / G)}{\Leftarrow} B$, i.e., $A \stackrel{G}{\leftarrow} B$.

(Only if). Suppose $A \stackrel{G}{\leftarrow} B$, that is, there exists a supervisor $S$ such that $A \stackrel{(S / G)}{\Leftarrow} B$. Let $E^{S}$ denote the edge set of $(S / G)$ and construct the required subprocess $G^{\prime}=\left(V^{\prime}, E^{\prime}\right)$ according to

$$
V^{\prime}=r_{S / G}(B)
$$

and

$$
E^{\prime}=\left\{(v, \sigma, u) \in E^{S}\right.
$$

$(S / G)$

Clearly, $B \subseteq V^{\prime}$. It remains to show that $G^{\prime}$ satisfies conditions (b1)-(b3). Indeed, $A \stackrel{(S / G)}{\models} B$ and thus conditions (a2) and (a3), with $(S / G)$ instead of $G$, imply the satisfaction of (b1) and (b3) w.r.t. $G^{\prime}$. Further, since $E_{u} \subseteq E^{S}$ it follows by (4.3) that $G^{\prime}$ is realizable, satisfying condition (b2).

Proposition 4.1 provides necessary and sufficient conditions for the solvability of the Weak Attraction Problem (WAP), namely given a process $G=(V, E)$ and subsets $A \subseteq B \subseteq V$, verify whether WAP is solvable or not. Notice that if $\Sigma_{u}=\varnothing \quad$ (i.e., every edge of $G$ is controlled) then WAP is solvable iff each state in $B$ is connected to a state in $A$. However, if the former condition does not hold (i.e., $\Sigma_{u} \neq \varnothing$ ) then WAP is not necessarily solvable even if the latter condition is satisfied.

So far we considered only $E_{u}$-invariant subsets of $V$ as candidates for weak attractors. Clearly, this is a necessary condition. Suppose, however, that we are given two subsets $A$ and $B$, such that $A \subseteq B \subseteq V$ and $A$ is not $E_{u}$-invariant. An interesting question is whether there exists a subset $A^{\prime} \subseteq A$ such that $A^{\prime} \stackrel{G}{\leftarrow} B$. That is, find (if exists) a subset $A^{\prime}$ of $A$ for which a supervisor $S$ 
can be synthesized, so that from each initial state $v \in B$, the closed-loop process $(S / G)$ reaches $A^{\prime}$ in finite number of state transitions and remains in $A^{\prime}$.

The following intuitive proposition states that the problem above is solvable iff the maximal $E_{u}$-invariant subset of $A$ is a weak attractor for $B$. The fact that every subset $A \subseteq V$ contains a unique maximal $E_{u}$-invariant subset, denoted $A^{\#}$, can be easily verified (cf. Ramadge and Wonham 1987 b, sec. 7).

\section{Proposition 4.2}

Let $G=(V, E), \varnothing \neq A \subseteq B \subseteq V$. There exists a subset $A^{\prime} \subseteq A$ such that $A^{\prime} \leftarrow B$ if and only if $A^{\#} \stackrel{G}{\leftarrow} B$.

\section{Proof}

(If). Trivially, since $A^{\#} \subseteq A$.

(Only if). Suppose $A^{\#}$ is not a weak attractor for $B$ w.r.t. $G$, and assume, towards a contradiction, that $A^{\prime} \stackrel{G}{\leftarrow} B$ for some $A^{\prime} \subseteq A$. It follows by proposition 4.1 , that $G=(V, E)$ contains a subprocess $G^{\prime}=\left(V^{\prime}, E^{\prime}\right)$ such that $B \subseteq V^{\prime}$ and conditions (b1)-(b3) are satisfied. That is, $G^{\prime}$ is realizable and $A^{\prime}$-connected, and $G^{\prime}-A^{\prime}$ is acyclic. On the other hand, every weak attractor is necessarily $E_{u}$-invariant, implying the inclusion $A^{\prime} \subseteq A^{\#}$. These two conclusions imply the satisfaction of conditions (b1)-(b3) also w.r.t. $A^{\#}$, namely $G^{\prime}$ is $A^{\#}$-connected and $G^{\prime}-A^{\#}$ is acyclic. This contradicts our assumption that $A^{\#}$ is not a weak attractor for $B$.

An effective computation of $A^{\#}$ is provided in Ramadge and wonham $1987 \mathrm{~b}$, sec. 7, based on a fixed point characterization of $A^{\#}$. The verification whether $A^{\#}$ is a weak attractor for $B$ can be accomplished by using the algorithm presented in section 5 . 


\subsection{Region of weak attraction}

Let $G=(V, E)$ be a process. In a previous section we showed that for every $E$-invariant subset $A$ there is a (unique) maximal subset $B$ for which $A \stackrel{G}{\Leftarrow} B$, and thus the notion of the region of strong attraction is well defined. In this section we examine whether an analogous notion can be defined for weak attraction. That is, given a nonempty subset $A \subseteq V$, we want to know whether the class of subsets that are weakly attracted by $A$ is closed under set union, and hence has a maximal element.

Let $A$ be $E_{u}$-invariant and define the class of subsets $W_{G}(A)$ according to

$$
W_{G}(A)=\{B \subseteq V \mid A \subseteq B \text { and } A \stackrel{G}{\leftarrow} B\}
$$

\section{Proposition 4.3:}

Let $A$ be an $E_{u}$-invariant subset of $V$. Then the class $W_{G}(A)$ is nonempty and closed under set union.

\section{Proof}

Let $G=(V, E)$ and let $A$ be an $E_{u}$-invariant subset of $V$. The nonemptiness of $W_{G}(A)$ follows from the fact that $A$ is a weak attractor for itself.

Let $B_{1}, B_{2} \in W_{G}(A)$, that is, $A \stackrel{G}{\leftarrow} B_{1}$ and $A \stackrel{G}{\leftarrow} B_{2}$. We have to show that $A \stackrel{G}{\leftarrow}\left(B_{1} \cup B_{2}\right)$. Recall that according proposition 4.1 there exist subprocesses $G_{1}=\left(V_{1}, E_{1}\right)$ and $G_{2}=\left(V_{2}, E_{2}\right)$ such that $B_{1} \subseteq V_{1}, B_{2} \subseteq V_{2}$ and each of $G_{1}$ and $G_{2}$ satisfies conditions (b1)-(b3). It is quite natural to verify whether $A$ is a weak attractor for $B_{1} \cup B_{2}$ by considering first the union of $G_{1}$ and $G_{2}$, i.e., the process $G_{12}=\left(V_{1} \cup V_{2}, E_{1} \cup E_{2}\right)$, and checking whether it satisfies the conditions of proposition 4.1. Indeed, it is easily seen that the $A$-connectivity and realizability properties are preserved under union of processes. Thus the process $G_{12}$ satisfies conditions (b1) 
and (b2). However, $G_{12}-A$ is not necessarily acyclic and thus condition (b3) is not necessarily satisfied. Nevertheless, in the following we show that $G_{12}$ contains a subprocess $P$ satisfying conditions (b1)-(b3).

Let $C=u_{0}, u_{1}, \cdots, u_{k}, u_{k}=u_{0}$ be a cycle of $G_{12}-A$, and let $s=e_{1}, e_{2}, \cdots, e_{k}$ be the path corresponding $C$, i.e., the end states of an edge $e_{i}$ are $u_{i-1}$ and $u_{i}$. For simplicity we assumed here that there are no parallel edges in $G$ and thus the path $s$ is uniquely defined by the cycle $C$. Since every edge of $C$ is in $E_{1} \cup E_{2}$, and $G_{1}-A$ and $G_{2}-A$ are acyclic, there exists at least one edge, say $e_{i}$, satisfying the condition that

$$
e_{i} \in E_{1} \quad \text { and } \quad e_{i+1} \in E_{2}-E_{1} .
$$

It is clear that the removal of the edge $e_{i+1}$, as well as the deletion of any other edge of the cycle $C$, opens $C$. However, the exclusive consequence of the deletion of $e_{i+1}$ is the opening of $C$, without affecting conditions (b1) and (b2). That is, $C$ is no longer a cycle of $G_{12}-e_{i+1}-A$, but $G_{12}-e_{i+1}$ is still realizable and $A$-connected. This argument is proved as follows. Let $u_{i}$ be the end state of $e_{i}$ and the start state of $e_{i+1}$. Since $e_{i} \in E_{1}$ and $e_{i+1} \in E_{2}$ it is clear that $u_{i}$ is a state in $G_{1}$ and $G_{2}$. Now, the deletion of $e_{i+1}$ might affect only the $A$-connectivity of $u_{i}$ and those states which are connected to $A$ through $u_{i}$. However, since $e_{i+1} \notin E_{1}$ and $G_{1}$ is $A$-connected, there exists a path from $u_{i}$ to $A$ which does not traverse $e_{i+1}$. Consequently, $G_{12}-e_{i+1}$ is $A$ connected and condition (b2) is satisfied w.r.t. $G_{12}-e_{i+1}$. As regards condition (b2), the realizability of $G_{12}-e_{i+1}$ follows from the fact that $e_{i+1}$ must be a controlled edge. Otherwise it would have followed from (4.5) that $G_{1}$ does not include the uncontrolled edge $e_{i+1}$ emanating from the state $u_{i} \in V_{1}$, contradicting our assumption that $G_{1}$ is realizable. So we have proved that $G_{12}-e_{i+1}$ is realizable, $A$-connected and does not include the cycle $C$.

The procedure above is repeated for each cycle of $G_{12}-A$ (the number of these repetitions is bounded by $|E|$ ). By the argument of the preceding paragraph it is clear that at termination the resulting subprocess $P$ satisfies conditions (b1)-(b3). So, by proposition 4.1, 


$$
A \stackrel{G}{\leftarrow}\left(B_{1} \cup B_{2}\right) .
$$

Since $W_{G}(A)$ is finite and closed under set union it follows that $W_{G}(A)$ contains a unique supremal element w.r.t. inclusion, denoted $\Omega_{G}(A)$ and called the region of weak attraction of $A$ w.r.t. $G$. If $A$ is not $E_{u}$-invariant we say that $\Omega_{G}(A)=\varnothing$. Further, if $\Omega_{G}(A)=V$ we say that $A$ is a global weak attractor w.r.t. $G$, denoted $A \stackrel{G}{\leftarrow}$.

It is easily seen that

$$
\Lambda_{G}(A) \subseteq \Omega_{G}(A)
$$

for every $A \subseteq V$.

\section{Computation of $\Omega_{G}(A)$}

Fix $G=(V, E), \varnothing \neq A \subseteq V$. In this section we propose an algorithm for the computation of the region of weak attraction $\Omega_{G}(A)$. A by-product of this algorithm is a subprocess of $G$ satisfying conditions (b1)-(b3). Further, the question of whether $A$ is a weak attractor for a subset $B \supseteq A$ is equivalent to the question of whether $B \subseteq \Omega_{G}(A)$. Thus, the algorithm provides a constructive method for verifying weak attraction.

Throughout this section we assume that $A$ is $E_{u}$-invariant, for otherwise $\Omega_{G}(A)=\varnothing$.

We derive now an intuitive consequence of proposition 4.1 concerning the region of weak attraction. Since, by definition, $A \stackrel{G}{\leftarrow} \Omega_{G}(A)$, it follows by proposition 4.1 that there exists a subprocess $G^{\prime}=\left(V^{\prime}, E^{\prime}\right)$ of $G$ such that $\Omega_{G}(A) \subseteq V^{\prime}$ and $G^{\prime}$ satisfies condition (b1)-(b3). Moreover, $G^{\prime}$ must satisfy the condition that $V^{\prime}=\Omega_{G}(A)$. Otherwise the process $G^{\prime}$ would have been a contradiction to the assumption that $\Omega_{G}(A)$ is the largest subset for which $A$ is a weak attractor. 
We have proved:

\section{Proposition 5.1}

Let $G^{\prime}=\left(V^{\prime}, E^{\prime}\right) \subseteq G$ be a subprocess such that $\Omega_{G}(A) \subseteq V^{\prime}$. If $G^{\prime}$ satisfies (b1)-(b3), then

$$
V^{\prime}=\Omega_{G}(A)
$$

The subprocess $G^{\prime}=\left(V^{\prime}, E^{\prime}\right)$ in proposition 5.1 is not necessarily unique. However, its state set $V^{\prime}$ is unique. The result of the algorithm below for computing $\Omega_{G}(A)$ is a subprocess of $G$ that satisfies conditions (b1)-(b3) and whose state set is $\Omega_{G}(A)$. But first we need the following definition.

Let $G^{\prime}=\left(V^{\prime}, E^{\prime}\right) \subseteq G=(V, E)$ be a process satisfying conditions (b1)-(b3), that is, $G^{\prime}$ is realizable and $A$-connected and $G^{\prime}-A$ is acyclic. We say that a state $v \in V-V^{\prime}$ is $G^{\prime}$-attractable iff $v$ is a predecessor of a state in $V^{\prime}$ and every uncontrolled edge of $G$ leaving $v$ ends in $V^{\prime}$, that is, $v \in V-V^{\prime}$ is $G^{\prime}$-attractable iff

(i) $\quad(\exists(v, \sigma, u) \in E) \quad u \in V^{\prime}$; and

(ii) $\left(\forall(v, \sigma, u) \in E_{u}\right) \quad u \in V^{\prime}$.

Now we are ready for the following

\section{ALGORITHM}

Input : A process $G=(V, E)$ and a subset $A \subseteq V$.

Output: A subprocess $P$ whose state set is $\Omega_{G}(A)$.

(1) Let $P_{o} \triangleq\left(U_{o}, D_{o}\right)=\langle A\rangle_{G}, j:=0$.

(2) If there are no $P_{j}$-attractable states in $V-U_{j}$ then $P=P_{j}$, stop. 
(3) Let $v \in V-U_{j}$ be a $P_{j}$-attractable state.

Define $P_{j+1} \triangleq\left(U_{j+1}, D_{j+1}\right)$ as

$$
\begin{gathered}
U_{j+1}=U_{j} \cup\{v\} \\
D_{j+1}=D_{j} \cup\left\{(v, \sigma, u) \in E \mid u \in U_{j}\right\} . \\
j:=j+1, \quad \text { go to (2). }
\end{gathered}
$$

That is, the construction of a subprocess $G^{\prime}$ whose state set is $\Omega_{G}(A)$ is started from the subprocess $P_{o}$ induced by $A$ (step (1)). Then, in each iteration $j$ a new subprocess $P_{j+1}$ is constructed (step (3) ) from $P_{j}$ by adding a $P_{j}$-attractable state $v$ together with every edge going from $v$ to a state of $P_{j}$. This procedure terminates when $P_{j}$ has no more attractable states (step (2)).

Since in each iteration the state set of $P_{j}$ increases by one state, the number of iterations is bounded by $|V|$. Further, it is easily seen that the verification of step (2), namely that there exists a $P_{j}$-attractable state in $V-U_{j}$, is of complexity $O(|\Sigma||V|)$. Thus, the complexity of the algorithm above is $O\left(|\Sigma||V|^{2}\right)$.

The correctness of this algorithm is formally stated in the following theorem.

\section{Theorem 5.1}

Let $P=(U, D)$ be the process obtained in step (2). Then

(i) $P$ satisfies conditions (b1)-(b3).

(ii) $U=\Omega_{G}(A)$.

For the proof of Theorem 5.1 we need the three following propositions. Intuitively, the first proposition states that $A$ is a weak attractor for the state set of each process $P_{j}$. Formally, we have 
the following

\section{Proposition 5.2}

For every iteration $j$, the process $P_{j}$ satisfies conditions (b1)-(b3), that is, $P_{j}$ is realizable and $A$-connected and $P_{j}-A$ is acyclic.

\section{Proof of Proposition 5.2}

This proposition is proved by induction on the number of iterations. Let $P_{o} \triangleq\left(U_{o}, D_{o}\right)=\langle A\rangle_{G}$, i.e., $P_{o}$ is the process induced by $A$. Trivially we have that $P_{o}$ is $A$ connected and that $P_{o}-A$ is acyclic. The realizability of $P_{o}$ follows from the facts that $A$ is $E_{u^{-}}$ invariant and that $D_{o}$ contains every edge of $G$ whose end points are in $A$. Thus, every uncontrolled edge leaving a state in $A$ is in $D_{o}$.

Suppose the proposition holds for the iteration $j$, and let $v \in V-U_{j}$ be a $P_{j}$-attractable state and $P_{j+1}=\left(U_{j+1}, D_{j+1}\right)$ be the process as defined in step (3). We have to show that $P_{j+1}$ satisfies conditions (b1)-(b3).

As regards (b1) and (b2) (A-connectivity and realizability), it is enough to consider the state $v$ in $P_{j+1}$ because according to the inductive hypothesis $P_{j}$ is $A$-connected and realizable. Indeed, since $v$ is $P_{j}$-attractable it follows that $v$ is a predecessor of a state of $P_{j}$, say $u \in U_{j}$. Furthermore, the assumption that $P_{j}$ is $A$-connected implies the existence of a path from $u$ to a state in $A$, as well as from $v$. So $P_{j+1}$ is $A$-connected. Next, the $P_{j}$-attractability of $v$ implies that every uncontrolled edge leaving $v$ ends in $U_{j}$, i.e., every uncontrolled edge leaving $v$ is included in $D_{j+1}$. So $P_{j+1}$ is realizable.

Finally, $P_{j+1}-A$ is acyclic because $P_{j}-A$ is acyclic and every edge added to $D_{j}$, in order to form $D_{j+1}$, is directed from $v$ to a state in $U_{j}$. Thus we conclude that $P_{j+1}$ satisfies conditions (b1)-(b3). 
The second proposition clarifies the role of attractable states.

\section{Proposition 5.3}

Let $P=(U, D) \subseteq G$ such that $A \subseteq U$ and $P$ satisfies conditions (b1)-(b3). Then every $P$ attractable state $v \in V-U$ is in the region of weak attraction of $A$, i.e.,

$$
v \in \Omega_{G}(A) .
$$

\section{Proof of Proposition 5.3}

Let $P=(U, D) \subseteq G$ such that $A \subseteq U$ and $P$ satisfies (b1)-(b3), and let $v \in V-U$ be $P$ attractable. Define the process $P^{\prime}=\left(U^{\prime}, D^{\prime}\right)$ according to

$$
U^{\prime}=U \cup\{v\}
$$

and

$$
D^{\prime}=D \cup\{(v, \sigma, u) \in E \mid u \in U\}
$$

It is easily seen that $P^{\prime}$ results from $P$ after one iteration of the algorithm above. Thus, by proposition 5.2 it follows that $P^{\prime}$ satisfies conditions (b1)-(b3). Further, by corollary 4.1 we get that $A$ is a weak attractor for $U^{\prime}$. Since by the definition of the region of weak attraction $U^{\prime} \subseteq \Omega_{G}(A)$ it follows that $v \in \Omega_{G}(A)$, which completes our proof.

The final proposition required for the proof of theorem 5.1 characterizes the class of subprocesses of $G$ whose state set is the region of weak attraction of $A$. 


\section{Proposition 5.4}

Let $P=(U, D) \subseteq G$ such that $A \subseteq U$ and $P$ satisfies conditions (b1)-(b3). Then

$$
U=\Omega_{G}(A)
$$

if and only if there are no $P$-attractable states in $V-U$.

\section{Proof of proposition 5.4}

Let $P=(U, D) \subseteq G=(V, E)$ such that $A \subseteq U$ and $P$ satisfies conditions (b1)-(b3).

(If). Let $X$ denote the set of all states in $V-U$ that are predecessors of a state in $U$, i.e.

$$
X=\{x \in V-U \mid(\exists(x, \sigma, u) \in E) \quad u \in U\} ，
$$

and suppose that every state in $X$ is not $P$-attractable. We have to prove that $U=\Omega_{G}(A)$.

First notice that $P$ satisfies conditions (b1)-(b3) and thus, by corollary $4.1, U \subseteq \Omega_{G}(A)$. For the reverse inclusion we shall show first that none of the states in $X$ can be in the region of weak attraction of $A$, i.e.,

$$
X \cap \Omega_{G}(A)=\varnothing
$$

For this let $x_{1} \in X \subseteq V-U$ and suppose, towards a contradiction, that $x_{1} \in \Omega_{G}(A)$. According to proposition 4.1, if $\left(U \cup\left\{x_{1}\right\}\right) \subseteq \Omega_{G}(A)$ then there exists a subprocess $G^{\prime}=\left(V^{\prime}, E^{\prime}\right)$ of $G$ such that $\left(U \cup\left\{x_{1}\right\}\right) \subseteq V^{\prime}$ and $G^{\prime}$ satisfies conditions (b1)-(b3). Since none of the states in $X$ is $P$-attractable then there exists an edge $e_{1}=\left(x_{1}, \sigma, v_{1}\right) \in E_{u}$ such that $v_{1} \notin U$. The edge $e_{1}$ is uncontrolled and thus $e_{1}$, as well as $v_{1}$, must be included in $G^{\prime}$. Otherwise $G^{\prime}$ could not be realizable (condition (b2)). Moreover, $G^{\prime}$ is $A$-connected and thus it must contain a trajectory from $v_{1}$ to a state in $U$ (notice that every state in $U$ is connected to $A$ ). Now, since $v_{1} \notin U$ there are two possibilities: either $v_{1} \in V-U-X$ or $v_{1} \in X$.

(i) If $v_{1} \in V-U-X$ then every trajectory of $G^{\prime}$ from $v_{1}$ to a state in $U$ must include at least one state in $X$ (this is because every predecessor of a state in $U$ is in $X$ ). Let $t$ be such a 
trajectory, namely a trajectory connecting $v_{1}$ to $U$, and let $x_{2}$ be the first state in $X$ traversed by $t$. Subsequently, denote by $t_{1}$ the subtrajectory of $t$ connecting $v_{1}$ to $x_{2}$, i.e., $t_{1}=v_{1}, \ldots, x_{2}$. Notice that none of the states of $t_{1}$ is a state in $U\left(\right.$ written $\left.t_{1} \cap U=\varnothing\right)$. Also, the condition $x_{2} \neq x_{1}$ must be satisfied in order that $G^{\prime}-A$ will be acyclic (otherwise $G^{\prime}-A$ will contain the cycle $\left.x_{1}, t_{1}\right)$.

(ii) If $v_{1} \in X$ then $x_{2}=v_{1}$ and $t_{1}$ is the empty trajectory.

Since $t_{1}$ is a trajectory of $G^{\prime}$ then $x_{2}$ is also a state of $G^{\prime}$. So we conclude that

$$
x_{1} \in V^{\prime} \quad \text { implies } \quad x_{2} \in V^{\prime}
$$

Following the argument of the previous paragraph we get that $G^{\prime}$ must contain a trajectory, say $t_{2}$, connecting $x_{2} \in X$ to $x_{3} \in X$, where $x_{3} \neq x_{2}, x_{3} \neq x_{1}$ and $x_{3} \in V^{\prime}$.

Continuing this procedure we end up with the following conclusions regarding the process $G^{\prime}: x_{1}$ is connected to $x_{2}$ by $t_{1}, x_{2}$ is connected to $x_{3}$ by $t_{2}, \cdots, x_{n-1}$ is connected to $x_{n}$ by $t_{n-1}, x_{n}$ is connected to $x_{j}, 1 \leq j \leq n$, by $t_{n}$ and

$$
x_{1}, x_{2}, \cdots, x_{n} \in V^{\prime}
$$

where $n$ is number of states in $X$ and $x_{i} \neq x_{j}, i \neq j$.

It is readily verified that the trajectory $t_{j}, t_{j+1}, \cdots, t_{n}$ forms a cycle in $G^{\prime}-A$ (notice that $A \subseteq U$ and $\left.t_{i} \cap U=\varnothing, 1 \leq i \leq n\right)$. Thus we conclude that the assumption $x_{1} \in V^{\prime} \subseteq \Omega_{G}(A)$ implies $X \subseteq V^{\prime}$. However, the requirement from $G^{\prime}$ to be $A$-connected implies the existence of a cycle in $G^{\prime}-A$, contradicting condition (b3). So

$$
X \cap \Omega_{G}(A)=\varnothing
$$

As regards the rest of the states in $V-U$; since every path from a state in $V-U-X$ to a state in $A$ must traverse at least one state in $X$ it is clear that

$$
(V-U-X) \cap \Omega_{G}(A)=\varnothing .
$$


>From (5.1) and (5.2) we get that $\Omega_{G}(A) \subseteq U$, which concludes the "if" part of this proof, i.e.,

$$
U=\Omega_{G}(A)
$$

(Only if). Suppose $U=\Omega_{G}(A)$ and assume there exists a state $v \in V-U$ such that $v$ is $P$ attractable. However, by proposition 5.3 it follows that $v \in \Omega_{G}(A)$, contradicting our assumption that $U$ is the region of weak attraction of $A$.

\section{Proof of Theorem 5.1}

Let $P=(U, D) \subseteq G=(V, E)$ be the process obtained in step (2) of the algorithm. By proposition 5.2 it is clear that $P$ satisfies conditions (b1)-(b3). Further, since every state in $V-U$ is not $P$-attractable (according to the condition of step (2) ) then by proposition 5.4

$$
U=\Omega_{G}(A)
$$

\section{Example}

Let $G=(V, E)$ be a process as displayed below: 


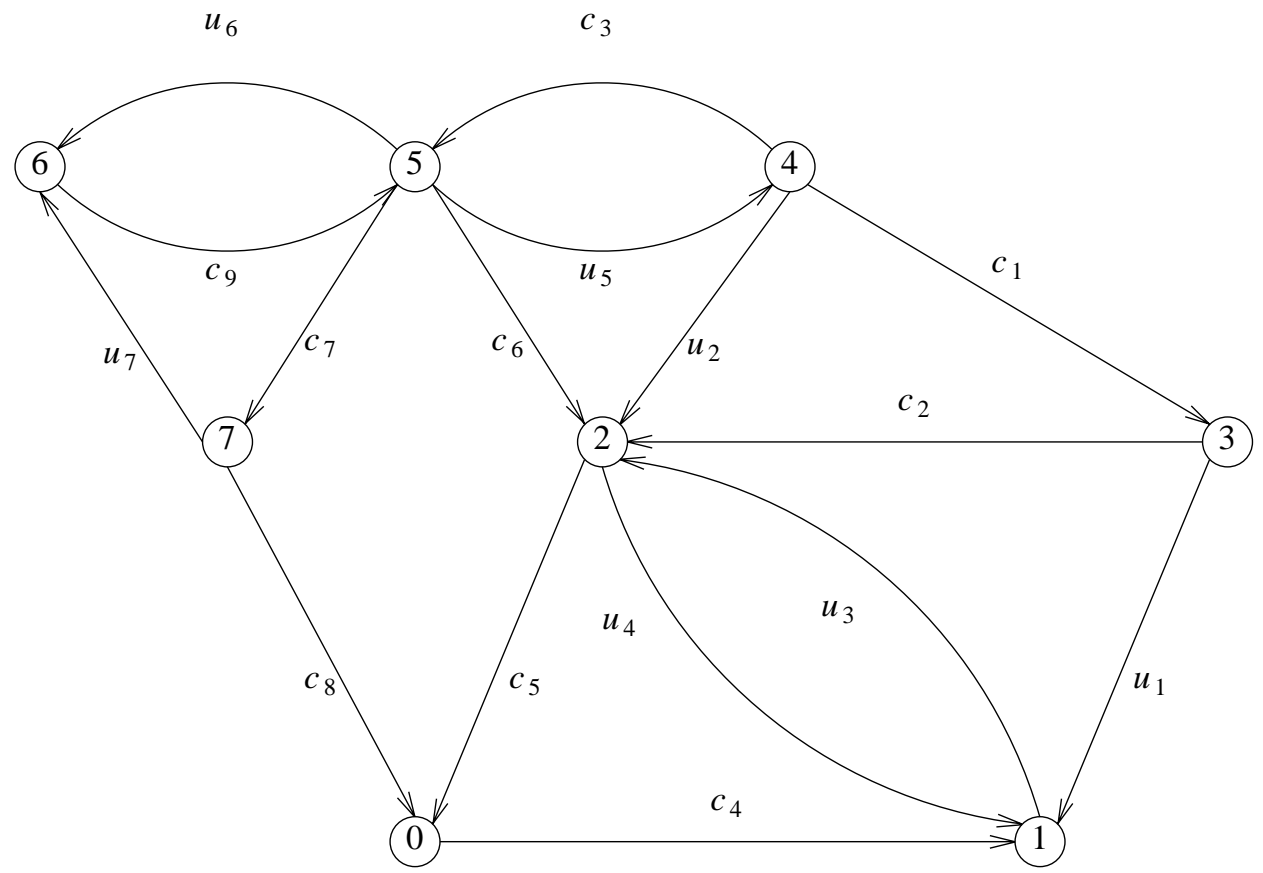

Figure 1.

The state set of $G$ is $V=\{0,1, \ldots, 7\}$, and the edge set is $E=\left\{u_{i}\right\} \cup\left\{c_{i}\right\}$. The edges denoted $u_{i}$ are uncontrolled while $c_{i}$ denotes a controlled edge.

First we comment that the subset $A_{1}=\{1,2\}$ cannot be a strong attractor for any subset of $V$ (since $A_{1}$ is not $E$-invariant). Nevertheless, $A_{1}$ is $E_{u}$-invariant and thus it has a potential to become a weak attractor (e.g., by the deletion of $c_{5}$ ).

Next we consider the subset $A=\{0,1,2\}$. Clearly $A$ is $E$-invariant, and if $B_{0}=A \cup\{3\}$ then $A$ is a strong attractor for $B_{0}$ w.r.t. $G$. It is easily seen that $B_{0}$ is the maximal subset of $V$ which is strongly attracted by $A$. That is, $\Lambda_{G}(A)=B_{0}$. Further, we remark that the region of strong attraction $\Lambda_{G}(A)$ can be computed in polynomial time by using the transitive closure of $G$ (see at the end of section 3).

We examine now the weak attraction problem, namely given two subsets $A, B$ of $V$, decide whether there exists a supervisor $S$ such that $A$ is a strong attractor for $B$ w.r.t. $(S / G)$. To this end, 
let $A=\{0,1,2\}, B_{1}=A \cup\{3,4\}$ and $B_{2}=A \cup\{7\}$. Recall that we defined weak $G$ attraction $A \leftarrow B$ as the possibility of driving $G$ (under control) from every initial state in $B$ to some state in $A$. Consequently, the deletion of the controlled edge $c_{3}$ implies $A \stackrel{G}{\leftarrow} B_{1}$. Furthermore, it is readily verified that the subprocess $\left\langle B_{1}\right\rangle_{G}$ (i.e. the subprocess induced by the states of $B_{1}$ ) satisfies the conditions of weak attraction, as stated in proposition 4.1.

As regards $B_{2}$, it can be shown that no subprocess of $G$, whose state set contains $B_{2}$, satisfies the conditions of proposition 4.1 (i.e., (b1)-(b3)). Thus we conclude that $A$ is not a weak attractor for $B_{2}$. Intuitively, this result can be explained as follows: Suppose $G$ is initialized at state $7 \in B_{2}$. Then either $G$ reaches state 0 (and then is captured in $A$ ) or it executes $u_{7}$ and reaches state 6 . Since the edge $u_{7}$ is uncontrolled (and thus cannot be removed from $G$ ) it follows that the edge $c_{9}$ must not be deleted from $G$. Otherwise the subset $A=\{0,1,2\}$ is not reachable from state 6 . However, the latter conclusion and the fact that $u_{6}$ is uncontrolled imply the existence of the cycle $C=6,5,6$. The cycle $C$ prevents the guaranteed attraction of state 7 to a state in $A$, i.e., if $G$ is initialized at state 7 then no control strategy can assure that $G$ (under control) will reach the subset $A$ after executing a finite number of state transitions.

The existence of a subprocess $G^{\prime}$ as required in proposition 4.1 can be effectively verified by using the algorithm of section 5 for computing the region of weak attraction. If we apply the algorithm to this example we obtain the following steps:

(i) Start with the subprocess $P_{o}=\langle A\rangle_{G}=\left(\{0,1,2\},\left\{u_{3}, u_{4}, c_{4}, c_{5}\right\}\right)$; (step (1)).

(ii) A candidate state for the next step is any predecessor of a state in $A$ which is $P_{o^{-}}$ attractable. Since the uncontrolled edges $u_{7}, u_{6}$ and $u_{5}$ lead to a state in $V-A$, none of the states 7 or 5 is $P_{o}$-attractable. Thus, choose for example state 3 and construct (step (3)) the subprocess 


$$
\begin{aligned}
P_{1} & =\left(\{0,1,2,3\},\left\{u_{3}, u_{4}, c_{4}, c_{5}, c_{2}, u_{1}\right\}\right) \\
& =\langle\{0,1,2,3\}\rangle_{G} .
\end{aligned}
$$

(iii) Only state 4 is $P_{1}$-attractable and thus construct (step (3) again) the subprocess

$$
\begin{aligned}
P_{2} & =\left(\{0,1,2,3,4\},\left\{u_{3}, u_{4}, c_{4}, c_{5}, c_{2}, u_{1}, u_{2}, c_{1}\right\}\right) \\
& =\langle\{0,1,2,3,4\}\rangle_{G} .
\end{aligned}
$$

(iv) There are no $P_{2}$-attractable states and thus the algorithm terminates; (step (2)).

By theorem 5.1 we conclude that $\Omega_{G}(A)=\{0,1,2,3,4\}=B_{1}$, and that $P_{2}$ satisfies the conditions of proposition 4.1. Based on $P_{2}$, a control pattern achieving weak attraction of $B_{1}$ by $A$ is readily synthesized (see the proof of proposition 4.1).

As was explained in the paragraph following proposition 5.1, the resulting process in step (2) is not unique. For example, if we had interchanged steps (ii) and (iii) we would have ended up with the process $P_{2}-c_{1}$. Nevertheless, the region of weak attraction of $A$ is yet $B_{1}$, since the state set of $P_{2}-c_{1}$ is $B_{1}$. This illustrates the consequence of proposition 4.3 , namely that the region of weak attraction is well defined.

Our intuitive conclusion that $A$ is not a weak attractor for $B_{2}$ is now an immediate consequence of the fact that $B_{2}$ is not a subset of $\Omega_{G}(A)$.

We end this example by pointing out the close relation between attraction properties and the problem of recovery from control failures. For example, suppose $A=\{0,1,2\}$ is the 'legal' state set of $V$, and that a control failure may cause $G$ to reach the illegal state 7 . Since $A$ is not a weak attractor for $B_{2}$, no control strategy can assure a guaranteed recovery (i.e., a guaranteed return of $G$ (under control) to a legal state in $A$ ) from this control failure. On the other hand,

\footnotetext{
G $A \leftarrow B_{1}$ implies the existence of a supervisor achieving guaranteed recovery from control failures causing $G$ to reach states 3 or 4 . Such a supervisor is readily synthesized by using the output of the algorithm in section 5 .
} 


\section{Conclusion}

The paper has presented the concept of strong attraction which plays a key role in the investigation of the following problems. The first one is the ability of a process to reach a set of target states from an arbitrary state and then remain there indefinitely. Another problem, which is closely related to the former, is the recovery from control failures. Finally, a special kind of asymptotic behavior of a process has been characterized as its minimal strong attractor. The first two problems were examined also under control, and an efficient procedure for synthesizing controllers that improve the attraction ability of processes has been proposed. The properties of such controllers and the extension of the above results for other representations of discrete event processes are interesting topics for further research. 


\section{REFERENCES}

Bhatia, N. P., and Szego, G. P., 1967, Dynamical systems: stability theory and applications. Lecture Notes in Mathematics 35, (Springer-Verlag, New York).

Brave, Y., and Heymann, M., 1989, EE PUB No. 714; see also Proc. 27th I.E.E.E. Conf. on Decision and Control, Austin, p. 1131.

Cho, H., and Marcus, S. I., 1987, preprint, Department of Electrical and Computer Engineering, University of Texas at Austin.

Cieslak, R., Desclaux, C., Fawaz, A., and Varaiya, P., 1988, I.E.E.E. Trans. autom. Control, 33, 249.

Even, S., 1979, Graph algorithms, (Computer Science Press).

Golaszewski, C. H., and Ramadge, P. J., 1989, On the control of real-time discrete event systems, Preprint.

Lin, F., and Wonham, W. M., 1987, System Control Group Report \#8701, Department of Elect. Eng., University of Toronto.

Ostroff, J. S., and Wonham, W. M., 1987, Proc. 26th I.E.E.E. Conf. on Decision and Control, Los Angeles, p. 656.

Ramadge, P. J., and Wonham, W. M., 1987 a, SIAM J. Control and Optimization, 25(1), 206.

Ramadge, P. J., and Wonham, W. M., 1987 b, SIAM J. Control and Optimization, 25(5), 1202.

Wonham, W. M., and Ramadge, P. J., 1987, SIAM J. Control and Optimization, 25(3), 637.

Yong, L., and Wonham, W. M., 1988, Proc. 27th I.E.E.E. Conf. on Decision and Control, Austin, Texas, p. 203. 